\title{
Attentional priming releases crowding
}

\author{
Árni Kristjánsson • Pétur Rúnar Heimisson • \\ Gunnar Freyr Róbertsson • David Whitney
}

Published online: 4 October 2013

(C) Psychonomic Society, Inc. 2013

\begin{abstract}
Views of natural scenes unfold over time, and objects of interest that were present a moment ago tend to remain present. While visual crowding places a fundamental limit on object recognition in cluttered scenes, most studies of crowding have suffered from the limitation that they typically involved static scenes. The role of temporal continuity in crowding has therefore been unaddressed. We investigated intertrial effects upon crowding in visual scenes, showing that crowding is considerably diminished when objects remain constant on consecutive visual search trials. Repetition of both the target and distractors decreases the critical distance for crowding from flankers. More generally, our results show how object continuity through between-trial priming releases objects that would otherwise be unidentifiable due to crowding. Crowding, although it is a significant bottleneck on object recognition, can be mitigated by statistically likely temporal continuity of the objects. Crowding therefore depends not only on what is momentarily present, but also on what was previously attended.
\end{abstract}

Keywords Crowding $\cdot$ Attention $\cdot$ Priming $\cdot$ Spatial vision

On a crowded basketball court, teammates must be found and opponents avoided. Team colors are key features remaining

\footnotetext{
Á. Kristjánsson $(\bowtie) \cdot P$. R. Heimisson · G. F. Róbertsson Faculty of Psychology, School of Health Sciences, University of Iceland, Reykjavik, Iceland e-mail: ak@hi.is

\author{
Á. Kristjánsson \\ Institute of Cognitive Neuroscience, University College London, \\ London, UK \\ D. Whitney \\ Department of Psychology, University of California, \\ Berkeley, CA, USA
}

constant while the players move around. While visual crowding may impair recognition of objects (and teammates) in such cluttered scenes (Levi, 2008; Pelli \& Tillman, 2008; Whitney \& Levi, 2011) the impact of crowding on object recognition is typically studied with individual static scenes. But views of natural scenes unfold over time, and objects of interest that were present a moment ago tend to remain present. No studies of crowding have systematically examined whether visual processing at one moment modulates recognition in subsequent cluttered scenes.

Effects of continued task relevance have been examined in visual search tasks (Maljkovic \& Nakayama, 1994). When the same visual search repeats, performance is improved (see Kristjánsson \& Campana, 2010; Lamy \& Kristjánsson, 2013, for reviews). Such priming effects are surprisingly large, modulating response times and accuracy by as much as $30 \%$. According to most accounts, priming reflects facilitated attention shifts (Becker; Chun \& Nakayama, 2000; Kristjánsson \& Nakayama; Lamy, Antebi, Aviani, \& Carmel, 2008). Priming effects have been observed during very brief presentation, excluding any role of response facilitation (Ásgeirsson, Kyllingsbæk, Kristjánsson, \& Bundesen, 2012; Sigurdardottir, Kristjánsson, \& Driver, 2008; Yashar \& Lamy, 2010). Additionally, priming effects are fundamentally bound to attentional selection (Brascamp, Blake, \& Kristjánsson, 2011; Goolsby \& Suzuki, 2001).

Yeshurun and Rashal (2010) reported increased identification accuracy from attentional precues for a target stimulus appearing among flankers (see also Dakin, Bex, Cass, \& Watt, 2009; Freeman \& Pelli, 2007; Strasburger, 2005). The cue reduced the critical distance for crowding indicating that attention reduces the spatial extent of crowding.

If attention modulates crowding, we should therefore expect attentional priming to modulate crowding. The prediction is straightforward: the more often the same search type repeats, interference from flankers upon search performance will be diminished. Here, we asked whether crowding from 
flankers is affected when the visual search targets to be attended and distractors to be avoided are constant between trials. Visual search trials were interspersed with crowding trials (see Fig. 1A). Observers located the oddly colored grating (red or green) and determined its orientation among three distractors of the other color (70-ms unmasked presentation). On $35 \%$ of the trials, task-irrelevant flankers appeared along with the search items (crowding trials) on the same radial line from fixation as the target and distractors (at variable distances from the target). Since priming typically builds up gradually and is larger the more often that search repeats (see, e.g., Kristjánsson, 2008; Maljkovic \& Nakayama, 1994), a second prediction was that effects upon crowding should decrease with more priming. If crowding is not a temporally independent process, then previous information (i.e., priming) about object properties should result in diminished crowding with increased priming. In Experiment 1 , the target and distractors swapped roles between trials. Since target and distractor repetition have dissociable effects upon attentional priming (Kristjánsson \& Driver, 2005, 2008; Lamy et al., 2008), in Experiments 2 and 3 we addressed the independent influence of target and distractor repetition on crowding.

\section{Experiment 1}

Method

Observers searched for an odd-color-out target among three distractors (Fig. 1). On $65 \%$ of the trials, only search items appeared, whereas four flankers appeared along with them on

a

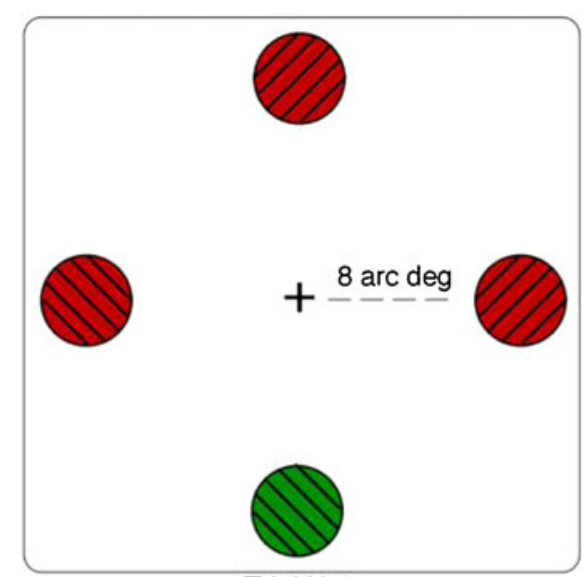

Trial N-2

b

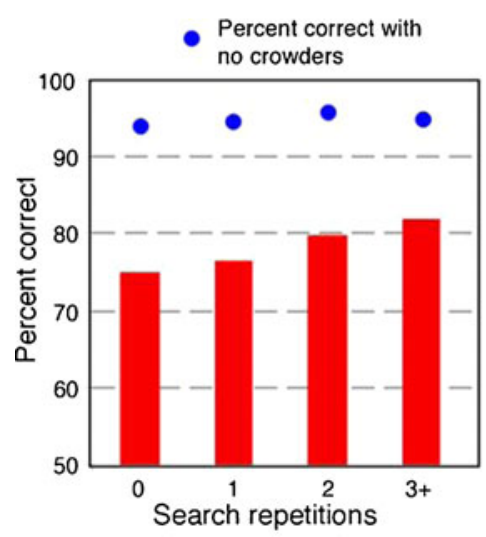

C

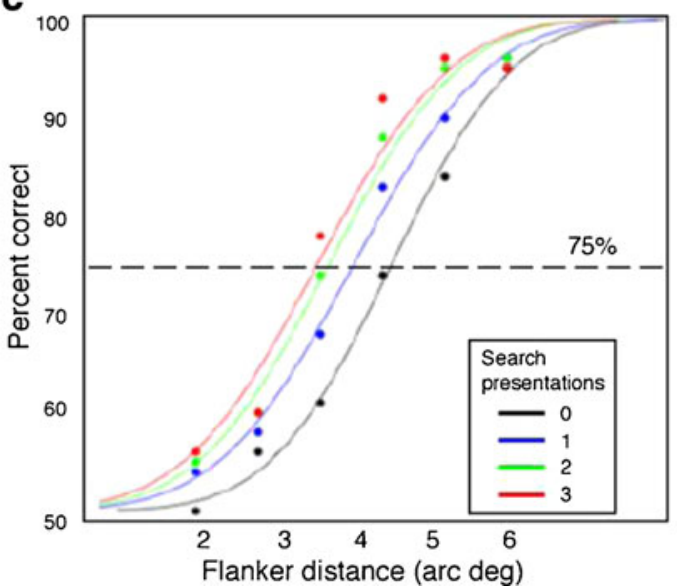

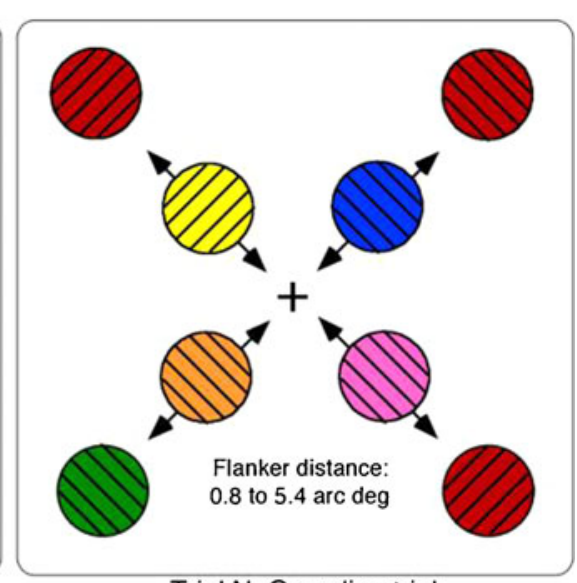

Trial N, Crowding trial

d

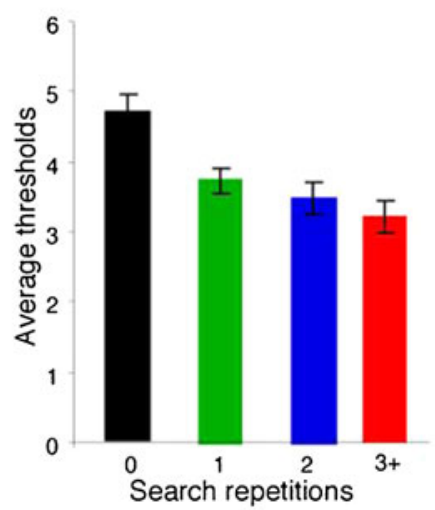

Fig. 1 Experimental design and results from Experiment 1. (A) Three possible consecutive search trials: on the right a crowding trial with flankers, following two search-only trials. The preceding trials share target and distractor colors with the crowding trial. The four flankers appear along with the search display on trial $N$. The items are not drawn to scale, and target and distractor locations were not predictable, except that they were always 90 radial degrees apart, whereas locations on the imaginary circle varied randomly. When flankers appeared (on $35 \%$ of the trials), they appeared on the same four radial axes from center as the target and distractors. (B) Average percents correct on crowding trials as a function of how often the same search type repeated. The dots denote the average percents correct on no-flanker trials. (C) Probit fits to the mean percent correct scores for the 12 observers, as a function of search repetition. (D) Average thresholds, estimated from the individual psychometric functions 
the other $35 \%$ (see Fig. 1A). We assessed how crowding was influenced by the number of similar search trials (with constant target and distractors) preceding each crowding display.

Participants A group of 12 naïve observers (eight female) participated in 600 trials. All had normal or corrected-tonormal vision.

Stimuli On search-only trials, four $1.8^{\circ}$ disks (viewing distance $=60 \mathrm{~cm}$ ) containing stripes oriented $\pm 45^{\circ}$ away from vertical appeared (see Fig. 1A). The target was the oddly colored item (either red [41 $\left.\mathrm{cdm}^{-2}\right]$ or green $\left[56 \mathrm{cdm}^{-2}\right]$ ), and the three other items of opposite color were distractors. Observers indicated by a keypress whether the target was oriented left or right from vertical. The search items appeared on an imaginary circle (radius from the center was $8^{\circ}$ ), with 90 radial degrees separating them. The phase on the imaginary circle varied randomly, so that the target and distractor locations were never predictable. On $35 \%$ of trials, randomly colored striped disks (flankers) appeared on the same radial axes as the search items, but closer to fixation (by $1.9^{\circ}, 2.7^{\circ}$, $3.5^{\circ}, 4.3,5.1^{\circ}$, or $5.9^{\circ}$ from the target, center-to-center, determined randomly for each trial), oriented randomly left or right. Crowding shows a tangential/radial asymmetry (Toet \& Levi, 1992), so the flankers were presented on the same radial axes as the search items. The flankers had random colors (blue [37 $\mathrm{cdm}^{-2}$ ], purple [39 $\mathrm{cdm}^{-2}$ ], yellow [98 $\left.\mathrm{cdm}^{-2}\right]$, and orange [62 $\left.\mathrm{cdm}^{-2}\right]$, one of each). The stimuli appeared on a 15-in., 85-Hz CRT display controlled by a G4 Macintosh. The displays were programmed in $\mathrm{C}$ utilizing the VisionShell functions.

Procedure A trial started with central fixation, followed 1, 000-1,400 ms later by the search display, appearing for $70 \mathrm{~ms}$ (unmasked). Observers judged by keypress whether the stripes on the oddly colored target were oriented left or right from vertical. To increase the number of consecutive identical search trials above chance, the probability of repetition was determined by $p($ rep $)=1-(24-\sqrt{ } N)$, where $N$ denotes the number of previous presentations of the same target color. On average, 64.2 crowding trials were presented following a search color change for each observer, 54.3 following one search repetition, 43.5 following two repetitions, and 51.6 following three or more repetitions. Observers were to ignore the flankers on crowding trials. A chinrest stabilized their heads throughout.

\section{Results}

The mean percents correct for crowding and no-crowding trials are presented in Fig. 1B. Performance was around $80 \%$ with flankers but $96 \%$ without [paired $t(11)=6.55, p<.001$ ].
More importantly, the more often a certain search was repeated, the smaller the crowding effect from the flankers. The effect of repetition upon accuracy was significant $[F(3,33)=$ $7.18, p<.001]$. The significant differences were between no and two $[t(11)=3.88, p=.003]$, no and three $[t(11)=5.69$; $p<.001]$, and one and three $[t(11)=4.63, p<.001]$ repetitions, while the differences between no and one and between three and four repetitions were not significant $(p>.05)$. We also tested for priming effects upon response times on the search-only trials finding a significant effect of search repetition $[F(3,33)=12.2, p=.001]$.

Any increase in performance from search repetition can reflect factors other than crowding. A hallmark of crowding is critical distance (Levi, 2008; Pelli \& Tillman, 2008; Whitney \& Levi, 2011), or how close to the target flankers must be to interfere with identification. We calculated center-to-center critical distance by plotting psychometric functions estimating $75 \%$ correct thresholds for the 4 repetition levels. Figure $1 \mathrm{C}$ shows the mean percents correct as a function of flankertarget distance, along with psychometric functions (dotted line $=$ collapsed subject threshold-performance). The critical distance diminished with increased search repetition (leftward-shift in psychometric functions). The more often that search repeated, the closer the flankers needed to be to interfere with target identification. Figure 1D shows the average thresholds estimated from individual psychometric functions. A series of $t$ tests of one versus four repetitions for each distance (Table 1) showed no effect at the smallest and largest flanker distances, indicating no vertical shift of the psychometric functions. Such differences were, however, significant for the three intervening distances showing that priming affects critical distance.

Experiment 1 shows that as search type repeats, crowding diminishes. Previous findings had indicated that priming influences attention shifts (Maljkovic \& Nakayama, 1994; Sigurdardottir et al., 2008). Our results therefore suggest that the more often a search target is repeated, the better it cues automatic shifts of attention (Kristjánsson, 2006), releasing search items from crowding from flankers.

\section{Experiment 2}

Although Experiment 1 showed that search repetition diminishes crowding, it did not distinguish between potential sources of the benefit: target repetition, distractor repetition, or both. Attentional priming occurs for both repeated distractors and targets (Geyer, Müller, \& Krummenacher, 2006; Lamy et al., 2008; Maljkovic \& Nakayama, 1994; Wang, Kristjánsson, \& Nakayama, 2005), independently and additively (Ásgeirsson, Kyllingsbæk, Kristjánsson \& Bundesen, 2012; Kristjánsson \& Driver, 2005, 2008). 
Table 1 T-tests assessing differences between one and four repetitions (Exp.1) and no-repetition versus repetition of target and distractors (Exps. 2 and 3 )

\begin{tabular}{|c|c|c|c|c|c|c|c|c|}
\hline \multicolumn{3}{|l|}{ Experiment 1} & \multicolumn{3}{|l|}{ Experiment 2} & \multicolumn{3}{|l|}{ Experiment 3} \\
\hline Target-Flanker Distance $\left({ }^{\circ}\right)$ & $t$ & $p$ & Target-Flanker Distance $\left(^{\circ}\right)$ & $t$ & $p$ & Target-Flanker Distance $\left(^{\circ}\right)$ & $t$ & $p$ \\
\hline 1.9 & 1.39 & .19 & 0.9 & 1.00 & .362 & 1.4 & 0.85 & .434 \\
\hline 2.7 & 1.69 & .12 & 1.8 & 1.04 & .347 & 2.1 & 1.56 & .180 \\
\hline 3.5 & 4.43 & .001 & 2.7 & 7.97 & $>.001$ & 2.8 & 3.77 & .013 \\
\hline 4.3 & 3.79 & .003 & 3.6 & 5.38 & .003 & 3.5 & 4.40 & .007 \\
\hline 5.1 & 3.00 & .012 & 4.5 & 3.85 & .012 & 4.2 & 2.73 & .041 \\
\hline \multirow[t]{2}{*}{5.9} & .81 & .43 & 5.4 & 0.83 & .44 & 4.9 & 2.68 & .044 \\
\hline & & & & & & 5.6 & 1.74 & .141 \\
\hline
\end{tabular}

In Experiment 1, 11 degrees of freedom; in Experiments 2 and 3, 5 degrees of freedom

Any effect from distractor repetition upon crowding would be of great interest, since it would suggest longerrange interactive effects across the visual field. Target repetition leads to a local benefit around the repeated feature, but an independent distractor effect could, by definition, not reflect local facilitation, but would have to involve inhibition of other locations.

In Experiment 2, we assessed the role of distractor repetition upon crowding, comparing it with target repetition effects to determine whether they interact or summate (see, e.g., Ásgeirsson et al., 2012; Kristjánsson, 2009; Lamy, Yashar, \& Ruderman, 2010). Again, observers judged the orientation of the odd-disk-out, but the possible target and distractor colors were four (red, green, blue, and yellow). This allowed us to investigate performance when only the target color repeated (e.g., red target and green distractors following a red target and blue distractors), when only the distractor color repeated (e.g., blue target and green distractors following a red target and green distractors), and when both or neither target and distractor colors repeated. To simplify the paradigm, role reversals of the target and distractors (see, e.g., Kristjánsson \& Driver, 2005, 2008) did not occur.

\section{Method}

Participants Six new observers participated in 2,000 trials (all naïve, with normal or corrected-to-normal vision).

Stimuli and procedure The possible target and distractor colors were now four (blue, red, yellow or green). The flankers were purple, orange, turquoise $\left(63 \mathrm{cdm}^{-2}\right)$, and brown $\left(31 \mathrm{cdm}^{-2}\right)$. The probability of each search type was now random, except for the constraint that role reversals did not occur. Otherwise methods were as in Experiment 1.
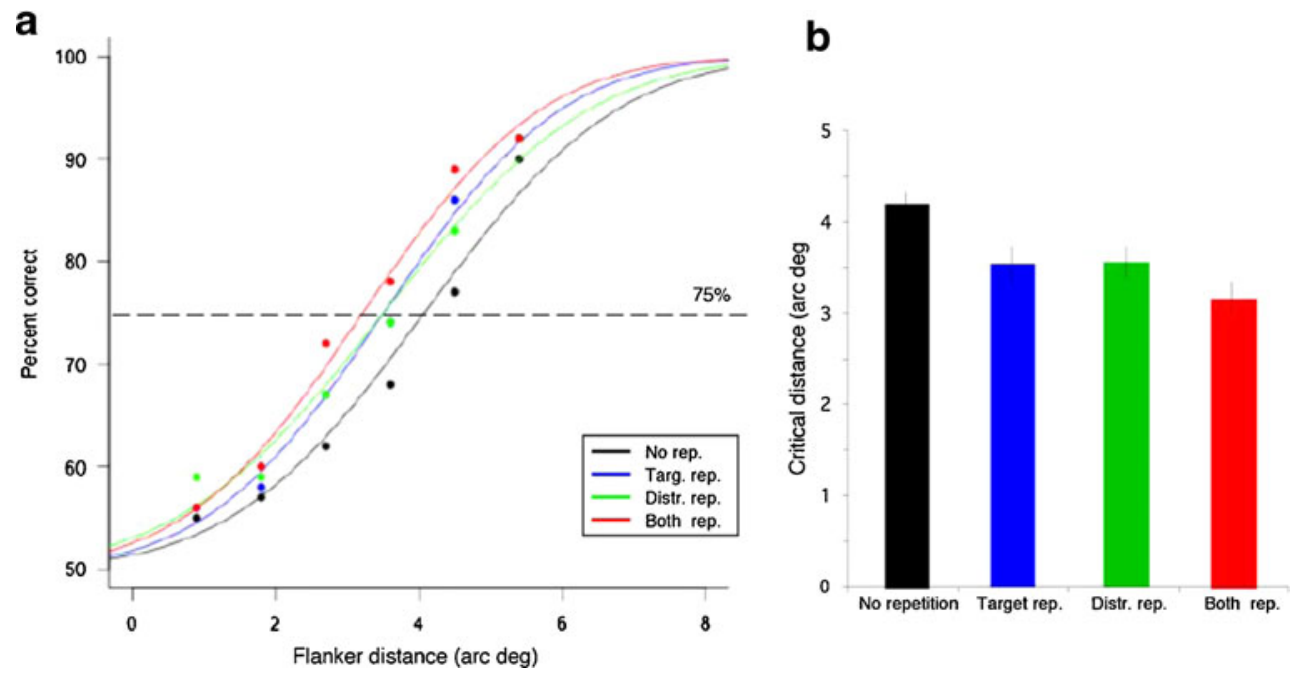

Fig. 2 Results of Experiment 2. (A) Probit fits to the mean percent correct scores. Differently colored lines denote the four different priming conditions: neither target nor distractor sets repeated, target color alone repeated, distractor color alone repeated, or both target and distractor colors repeated. (B)Critical distance, estimated from the $75 \%$-correct points on individual fitted functions 


\section{Results}

In Experiment 2, we measured the independent contributions of target versus distractor repetition and any additive or interactive effects of repetition. Figure 2 shows average performance as a function of repetition condition along with probit fits. Panel B plots the $75 \%$ thresholds estimated from individual psychometric functions.

Both target and distractor repetition diminished flanker crowding. As target or distractor color repeated, the critical distance decreased, and it was smallest when both were repeated (Fig. 2B). The main effects of both target $[F(1,5)=$ $7.97, p=.037]$ and distractor $[F(1,5)=7.16, p=.044]$ repetition on accuracy were significant but not the interaction $[F(1,5)=1.16, p=.33]$. Table 1 then confirms that the difference was not seen for the easiest and hardest conditions, indicating that the effect does not reflect a vertical shift in the psychometric functions.

Overall, the results suggest that the spatiotemporal continuity of both targets of interest and distractors to be avoided releases targets from crowding from flankers.

\section{Experiment 3}

Flankers typically induce larger crowding when they are more eccentric than the targets (Banks, Bachrach, \& Larson, 1977; Bouma, 1970; Farzin, Rivera, \& Whitney, 2009; Petrov, Popple, \& McKee, 2007). In Experiments 1 and 2, the flankers appeared closer to fixation than did the search items, which may not be maximally sensitive to crowding. Experiment 3 was a repeat of Experiment 2, except that the flankers were now more eccentric than the search items.
Method

Six observers participated (three new and three from Exp.2), all with normal or corrected-to-normal visual acuity. The eccentricity of the search items was $6.5^{\circ}$, while the flankers appeared at variable distances from them (from $1.4^{\circ}$ to $5.6^{\circ}$ ) on the same radial axes. The procedures were otherwise identical to those in the previous experiments.

Results

Figure 3 shows that with flankers more eccentric than the target, the critical distance was reduced by repeating both target and distractors, with the largest decreases in crowding measured when both target $[F(1,5)=11.98, p=.018]$ and distractor $[F(1,5)=7.59, p=.04]$ colors repeated. No interaction emerged $[F(1,5)=0.67, p=.45]$, again showing that the effects were independent. The $t$ tests in Table 1 show that the repetition effects did not reflect vertical shifts in the psychometric function. Experiment 3 supports the conclusion that crowding is affected by between-trial consistency of objects to attend to and distractors to reject, and confirms that this applies to flankers both more and less eccentric than the targets to be identified.

\section{General discussion}

Objects in visual scenes tend to remain present over time, and this mitigates the deleterious effects of clutter and improves recognition of objects that would otherwise be crowded. Attentional priming diminishes crowding from flankers through

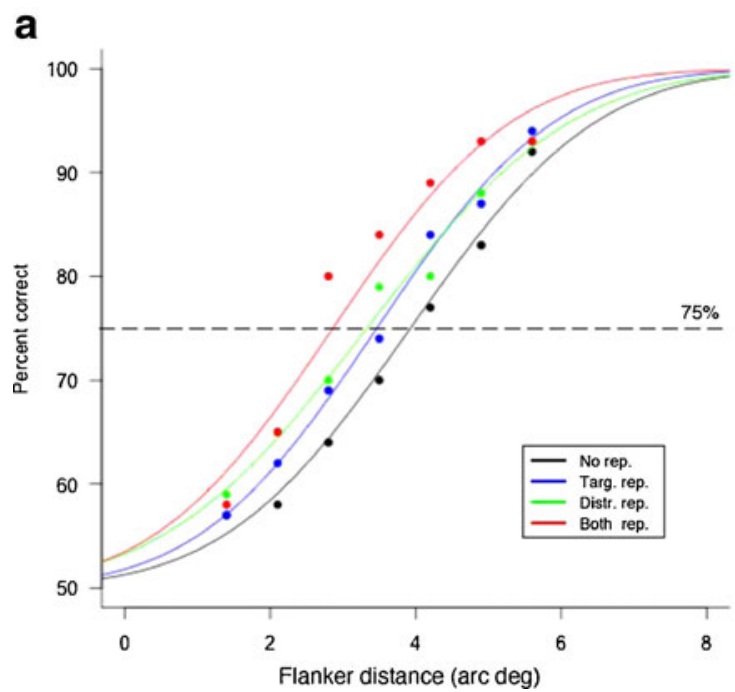

b

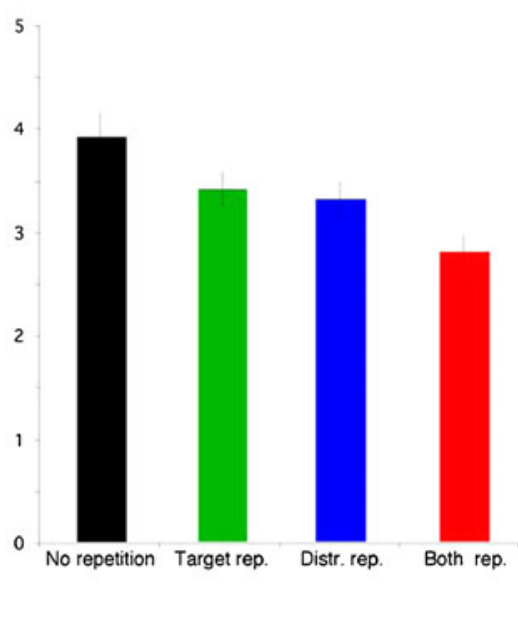

Fig. 3 Results of Experiment 3 (with flankers on the more eccentric, peripheral side of the search items, but otherwise presented in the same manner as in Exp. 2) 
influences of both target and distractor repetition, with the largest benefit when both repeat.

Perceptual crowding therefore depends not only on what is momentarily present on the retina, but on what was previously attended. The results cannot be attributed to target-flanker similarity or other modulations of crowding within single scenes (reviewed in Whitney \& Levi, 2011). Crowding modulation was solely due to an interaction over time reflecting benefits of regularity in sequential scenes.

Furthermore, the results here are not due to modulations of masking, generalized attention, or alerting. Priming modulated the critical spacing between flankers and targets, whereas masking and other phenomena are not specific to critical distance (Levi, 2008; Pelli \& Tillman, 2008). The benefit of priming was not an overall improvement (vertical shift in psychometric functions), but a leftward shift in the psychometric function, indicating access to finer resolution information at the target location. Second, crowding is stronger when the flankers are more eccentric than the target (Petrov et al., 2007), as in Experiment 3, in which the release of crowding was as strong as in the first two experiments, further cementing that attentional priming modulated crowding.

Whitney and Levi (2011) concluded that crowding occurs at various levels of visual processing, and therefore higher-level factors such as attention might affect it. There is growing support for this. Chakravarthi and Cavanagh (2007) investigated the crowding of polarity-reversing letter flankers. Crowding became phase independent at reversal rates of around $7.5 \mathrm{~Hz}$, perhaps reflecting the coarse temporal resolution of attention (e.g., Holcombe \& Cavanagh, 2001; Verstraten, Cavanagh, \& Labianca, 2000). Lower-level mechanisms such as masking or lateral inhibition did not explain the crowding. Yeshurun and Rashal (2010) assessed crowding at cued versus uncued locations, finding that attention reduced the spatial extent of crowding (see also Dakin et al., 2009; Freeman \& Pelli, 2007). Similarly, Strasburger (2005) concluded that impaired character recognition during crowding was caused by spatially imprecise focusing of attention.

The first experiment here is consistent with this role for attention in crowding, since the repeated target can serve as a cue. But the second and third experiments go beyond this conclusion, showing that repeated distractor sets also decrease crowding. We propose that the benefits reflect priming through temporal continuity of objects - that the visual system has a strong bias to favor the continuity of objects to attend to and others to avoid, using this to resolve crowding. Some authors have proposed that crowding may reflect spatial integration in the visual field, and that critical distance therefore reflects the size of integration zones (Parkes, Lund, Angelucci, Solomon, \& Morgan, 2001; Pelli, Palomares, \& Majaj, 2004). In the present context, we therefore speculate that attentional priming from spatiotemporal continuity may decrease the size of these zones, resulting in higher resolution at attended, or primed, locations. In sum, crowding, although it creates a significant bottleneck for object recognition, can therefore be strongly mitigated by statistically likely temporal continuity of the objects in natural scenes, creating clearer representations of objects such as teammates on a basketball court.

\section{References}

Ásgeirsson, Á., Kyllingsbæk, S., Kristjánsson, Á., \& Bundesen, C. (2012). The weight of the visual world is modified by recent experience: Modeling repetition priming in a partial report task [Abstract]. Journal of Vision, 12(9), 931. doi:10.1167/12.9.931

Banks, W. P., Bachrach, K. M., \& Larson, D. W. (1977). The asymmetry of lateral interference in visual letter identification. Perception \& Psychophysics, 22, 232-240.

Bouma, H. (1970). Interaction effects in parafoveal letter recognition. Nature, 226, 177-178.

Brascamp, J. W., Blake, R., \& Kristjánsson, Á. (2011). Deciding where to attend: Priming of pop-out drives target selection. Journal of Experimental Psychology: Human Perception and Performance, 37, 1700-1707. doi:10.1037/a0025636

Chakravarthi, R., \& Cavanagh, P. (2007). Temporal properties of the polarity advantage effect in crowding. Journal of Vision, 7(2), 113. 11.

Chun, M. M., \& Nakayama, K. (2000). On the functional role of implicit visual memory for the adaptive deployment of attention across scenes. Visual Cognition, 7, 65-81. doi:10.1080/135062800394685

Dakin, S. C., Bex, P. J., Cass, J. R., \& Watt, R. J. (2009). Dissociable effects of attention and crowding on orientation averaging. Journal of Vision, 9(11), 1-16. doi:10.1167/9.11.28. 28.

Farzin, F., Rivera, S. M., \& Whitney, D. (2009). Holistic crowding of Mooney faces. Journal of Vision, 9(6), 1-15. doi:10.1167/9.6.18. 18.

Freeman, J., \& Pelli, D. G. (2007). An escape from crowding. Journal of Vision, 7(2), 1-14. doi:10.1167/7.2.22. 22.

Geyer, T., Müller, H. J., \& Krummenacher, J. (2006). Cross-trial priming in visual search for singleton conjunction targets: Role of repeated target and distractor features. Perception \& Psychophysics, 68, 736749. doi:10.3758/BF03193697

Goolsby, B. A., \& Suzuki, S. (2001). Understanding priming of color singleton search: Roles of attention at encoding and "retrieval. Perception \& Psychophysics, 63, 929-944. doi:10.3758/ BF03194513

Holcombe, A. O., \& Cavanagh, P. (2001). Early binding of feature pairs for visual perception. Nature Neuroscience, 4, 127-128.

Kristjánsson, Á. (2006). Rapid learning in attention shifts: A review. Visual Cognition, 13, 324-362. doi:10.1080/13506280544000039

Kristjánsson, Á. (2008). "I know what you did on the last trial"-A selective review of research on priming in visual search. Frontiers in Bioscience, 13, 1171-1181.

Kristjánsson, Á. (2009). Independent and additive repetition priming of motion direction and color in visual search. Psychological Research, $73,158-166$.

Kristjánsson, Á., \& Campana, G. (2010). Where perception meets memory: A review of repetition priming in visual search tasks. Attention, Perception, \& Psychophysics, 72, 5-18. doi:10.3758/APP.72.1.5

Kristjánsson, Á., \& Driver, J. (2005). Priming in vision: Target repetition effects, context effects and role-reversal effects. Perception, 34(Suppl), 40c. 
Kristjánsson, Á., \& Driver, J. (2008). Priming in visual search: Separating the effects of target repetition, distractor repetition and role-reversal. Vision Research, 48, 1217-1232. doi:10.1016/j.visres.2008.02.007

Lamy, D., Antebi, C., Aviani, N., \& Carmel, T. (2008). Priming of popout provides reliable measures of target activation and distractor inhibition in selective attention. Vision Research, 48, 30-41. doi: 10.1016/j.visres.2007.10.009

Lamy, D. F., \& Kristjánsson, Á. (2013). Is goal-directed attentional guidance just intertrial priming? A review. Journal of Vision, 13(3), 14. doi:10.1167/13.3.14

Lamy, D., Yashar, A., \& Ruderman, L. (2010). A dual-stage account of inter-trial priming effects. Vision Research, 50, 1396-1401. doi:10. 1016/j.visres.2010.01.008

Levi, D. M. (2008). Crowding - an essential bottleneck for object recognition: A mini-review. Vision Research, 48, 635-654. doi:10.1016/j. visres.2007.12.009

Maljkovic, V., \& Nakayama, K. (1994). Priming of pop-out: I. Role of features. Memory \& Cognition, 22, 657-672. doi:10.3758/ BF03209251

Parkes, L., Lund, J., Angelucci, A., Solomon, J., \& Morgan, M. (2001). Compulsory averaging of crowded orientation signals in human vision. Nature Neuroscience, 4, 739-744.

Pelli, D. G., Palomares, M., \& Majaj, N. J. (2004). Crowding is unlike ordinary masking: Distinguishing feature integration from detection. Journal of Vision, 4(12), 1136-1169. doi:10.1167/4.12.12

Pelli, D. G., \& Tillman, K. A. (2008). The uncrowded window of object recognition. Nature Neuroscience, 11, 1129-1135. doi:10.1038/nn. 2187
Petrov, Y., Popple, A. V., \& McKee, S. P. (2007). Crowding and surround suppression: Not to be confused. Journal of Vision, 7(2), 1-9. doi: 10.1167/7.2.12. 12 .

Sigurdardottir, H. M., Kristjánsson, Á., \& Driver, J. (2008). Repetition streaks increase perceptual sensitivity in brief visual search displays. Visual Cognition, 16, 643-658. doi:10.1080/ 13506280701218364

Strasburger, H. (2005). Unfocused spatial attention underlies the crowding effect in indirect form vision. Journal of Vision, 5(11), 1024-1037. doi:10.1167/5.11.8. 8.

Toet, A., \& Levi, D. M. (1992). The two-dimensional shape of spatial interaction zones in the parafovea. Vision Research, 32, 1349-1357.

Verstraten, F. A. J., Cavanagh, P., \& Labianca, A. T. (2000). Limits of attentive tracking reveal temporal properties of attention. Vision Research, 40, 3651-3664. doi:10.1016/S0042-6989(00)00213-3

Wang, D., Kristjánsson, Á., \& Nakayama, K. (2005). Efficient visual search without top-down or bottom-up guidance. Perception \& Psychophysics, 67, 239-253. doi:10.3758/BF03206488

Whitney, D., \& Levi, D. M. (2011). Visual crowding: A fundamental limit on conscious perception and object recognition. Trends in Cognitive Sciences, 15, 160-168. doi:10.1016/j.tics.2011.02.005

Yashar, A., \& Lamy, D. (2010). Intertrial repetition affects perception: The role of focused attention. Journal of Vision, 10(14), 1-8. doi:10. 1167/10.14.3. 3.

Yeshurun, Y., \& Rashal, E. (2010). Precueing attention to the target location diminishes crowding and reduces the critical distance. Journal of Vision, 10(10), 1-12. doi:10.1167/10.10.16. 16. 